\title{
Use of Highly Discriminating Morphological Characters and Isozymes in the Study of Spanish Chestnut Cultivars
}

\author{
Santiago Pereira-Lorenzo', María Belén Díaz-Hernández, and Ana María Ramos-Cabrer \\ Universidad de Santiago de Compostela, Producción Vexetal, Escola Politécnica Superior, Campus \\ de Lugo, Lugo 27002, Spain
}

\begin{abstract}
AdDitional INDEX wORDs. Castanea sativa, classification, genetic diversity, morphology, multivariate analysis
ABstract. Morphological characters (six traits) and isozymes (four systems, five loci) were used to discriminate between Spanish chestnut cultivars (Castanea sativa Mill.) from the Iberian Peninsula. A total of 701 accessions (representing 168 local cultivars) were analyzed from collections made between 1989 and 2003 in the main chestnut growing areas: 31 were from Andalucía (12 cultivars), 293 from Asturias (65 cultivars), 25 from Castilla-León (nine cultivars), four from Extremadura (two cultivars) and 348 from Galicia ( 80 cultivars). Data were synthesized using multivariate analysis, principal component analysis, and cluster analysis. A total of 152 Spanish cultivars were verified: 58 cultivars of major importance and 94 of minor importance, of which 18 had high intracultivar variation. Thirty-seven cultivars were clustered into 14 synonymous groups. Six of these were from Galicia, one from Castilla-León (EI Bierzo), four from Asturias, one from Asturias and Castilla-León (EI Bierzo), and two from Asturias, Castilla-León (El Bierzo), and Galicia. The chestnut cultivars from Galicia and Asturias were undifferentiated in genetic terms, indicating that they are not genetically isolated. Overall, chestnut cultivars from southern Spain showed the least variation. Many (58\%) of Spanish cultivars produced more than 100 nuts/kg; removing this low market-value character will be a high priority. The data obtained will be of use in chestnut breeding programs in Spain and elsewhere.
\end{abstract}

Growers have selected chestnut cultivars since Roman times. Traditionally, chestnut stands are derived by grafting selected cultivars onto planted seedlings. In the Middle Ages huge plantations were established for forestry with the dual purpose of timber and nut production (Pereira-Lorenzo and Ramos-Cabrer, 2004). Some of these plantations can still be found in Tuscany (Italy), Galicia (Spain), Tras-Os-Montes (Portugal), and Aveyron and Cévennes (France). Monasteries established most of these extensive chestnut orchards.

There are 187 cultivars documented in Spain (Pereira-Lorenzo et al., 2001 a, 2001b). There is considerable need to define precisely chestnut cultivar variation for improved planting regimes and chestnut breeding. Breviglieri (1955) made the first comprehensive study of chestnut variation using morphological characteristics in the "Scheda Castanografica." Later, the International Union for the Protection of New Varieties of Plants (UPOV) published a chestnut guide for distinguishing chestnut cultivars and suggested some of the main discriminant characteristics (UPOV, 1988). Recently, some researchers have applied these guidelines to Spanish cultivars (Pereira-Lorenzoetal., 1996a; Ramos-Cabrer and Pereira-Lorenzo, 2005), Portuguese cultivars (Goulao et al., 2001), and to different chestnut species (Oraguzie et al., 1998).

Isozymes were the first markers used to study the genetic variation between chestnut cultivars (Pereira-Lorenzo et al., 1996b; Ramos-Cabrer and Pereira-Lorenzo, 2005; Sawano et al. 1984) and natural chestnut populations (Aravanopoulos et al., 2002; Pigliucci et al., 1990a, 1990b; Villani et al., 1991a, 1991b). Multivariate analysis has proved useful to discriminate genetic relationships within germplasm of Castanea sativa (Pereira-Lorenzo et al., 1996a, 1996b), C. dentata Borkh. (Huang et al., 1998; Oraguzie

Received for publication 18 Nov. 2005. Accepted for publication 10 May 2006.

1To whom reprint requests should be addressed; e-mail: spereira@lugo.usc.es et al., 1998), and C. pumila Mill. (Dane and Hawkins, 1999). A previous study of Spanish chestnuts showed a reduction in genetic variation (Ramos-Cabrer and Pereira-Lorenzo, 2005). However, no current data exist for the most important chestnut growing region, Asturias, in northern Spain. Therefore, the objectives here were to 1) classify Spanish chestnut cultivars for conservation and propagation purposes; and 2) determine the total variation of Spanish chestnuts. The data generated have significant implications for chestnut breeding programs.

\section{Materials and Methods}

Plant material. An inventory of some of the grafted cultivars included in this work and their locations has been published previously (Pereira-Lorenzo et al., 2001a). Accessions are a representative sample of cultivars cultivated in Spain. In 1999, a total of 293 accessions corresponding to 65 named cultivars were found in 145 different locations in 49 Councils from Asturias (Fig. 1; Table 1). Some of those cultivars were reported previously by Fernández-Lamuño (1984). Cultivars located in Asturias were compared with 348 accessions from 80 cultivars collected in Galicia from 1989-90, 31 accessions from 12 cultivars collected in Andalucía from 1996-2001, 25 accessions from nine cultivars collected in Castilla-León from 1996-2001, and four accessions from two cultivars collected in Extremadura from 1996-2001 (Pereira-Lorenzo et al., 1996a, 1996b, 2001a; Ramos-Cabrer and Pereira-Lorenzo, 2005). From each tree, nuts with burs were collected for 2 years, or more often, 3 years. Samples of catkins were also collected and preserved for further study.

Morphology AND PHENOLOGY. Traits were selected using UPOV (1988) criteria, some of which had previously been used to classify cultivars from Galicia (Pereira-Lorenzo et al., 1996a), Andalucía, Castilla-León, and Extremadura (Ramos-Cabrer and Pereira-Lorenzo, 2005). Three traits were quantitative: 1) nut 


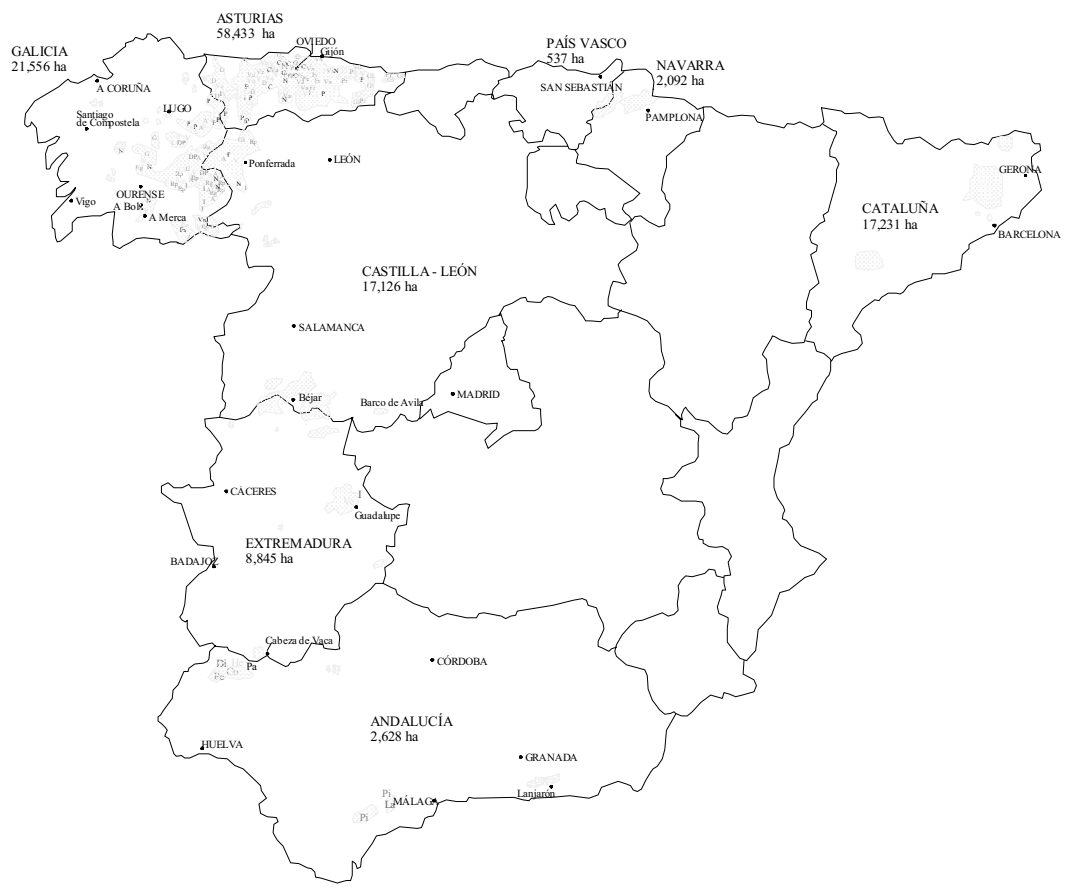

Fig. 1. Spanish chestnut areas where 168 cultivars were localized and 152 classified.

size expressed as number/kilogram (NNK) and weight of lateral nuts in grams (WLN), 2) nut width to length ratio (WLR $=100$ $\times$ width/length), and 3 ) percentage of divided nuts (PDN). Three traits were qualitative: 1) type of catkin: astaminate (CAS), brachystaminate (CBR), mesostaminate (CME), and longistaminate (CLO);2) size of bur spines: short(BTS), medium (BTM) and long (BTL); and 3) harvesting time: very early [VEA (15-25 Sept.)], early [EAR (26 Sept.-10 Oct.)], medium [MED (11-25 Oct.)], late [LAT (26 Oct.-11 Nov.)], and very late [VEL (after 11 Nov.)]. A new binomial variable was created for each class of qualitative trait, where the values 1 or 0 indicate presence or absence of the observed quality in the individual phenotype recorded (Kaufman and Rosseeuw, 1990). Thus, the three qualitative traits were split into 12 binomial variables.

IsOzYMEs. Four isozyme systems were used (Pereira-Lorenzo, 1996b): isocitrate dehydrogenase (IDH; EC 1.1.1.42), phosphoglucoisomerase (PGI; EC 5.3.1.9), malate dehydrogenase (MDH; EC 1.1.1.37), and shikimate dehydrogenase (SKDH; EC 1.1.1.25). An isotype was considered rare when its frequency was lower than 0.05 .

Analysis. To quantify genetic variation, data were analyzed using Fstat version 2.93 (Goudet, 1995) and Genepop version 3.3 (Raymond and Rousset, 1995) using the methods of Weir and Cockerham (1984) to estimate F statistics: the inbreeding coefficient $\left(\mathrm{F}_{\mathrm{is}}\right)$, the total fixation index $\left(\mathrm{F}_{\mathrm{it}}\right)$, and the proportion of differentiation among the populations $\left(\mathrm{F}_{\mathrm{st}}\right)$ (Wright, 1951).

Morphology variation was expressed as an average value for quantitative traits and as a frequency for qualitative traits. The relationships among accessions, using the 12 morphological traits and 13 isotypes, were examined with a principal components analysis (PCA) on the correlation matrix of the accession means [PRINCOMP of SAS (release 6.03; SAS Institute Inc., Cary, N.C.]. The Mahalanobis generalized distance ( $\mathrm{D}^{2}$ of Mahalanobis) (Goodman, 1972, 1973) was calculated as a dissimilarity measure between accessions from the standardized principal components (PCs) that had eigen values higher than one $\left(\lambda_{k}>\right.$ 1). The distance matrix was used in a cluster analysis (UPGMA) (Sneath and Sokal, 1973) to classify accessions and cultivars by their genetic similarity.

\section{Results}

Isozymes. Frequencies of isotypes for Asturian chestnut cultivars were similar to those previously found in Galicia (PereiraLorenzo et al., 1996a), except that there were fewer Mdh3-a and Idh-b isotypes. Idh-b was a rare isotype in Asturias and its frequency was closer to that found in middle and southern Spain (Castilla-León, Extremadura, and Andalucía) (Table 2). Isotype frequencies in Galicia are generally representative of those in the whole of Spain. Only Pgi-b can be considered a rare isotype in Galicia, Castilla-León, and Asturias, and is completely absent in Andalucía and Extremadura. The lower frequency of Idh-b found in Asturias was the main difference between Asturias and Galicia (representative of Spanish chestnuts in general). As reported previously (Pereira-Lorenzo et al., 1996b; Ramos-Cabrer and Pereira-Lorenzo, 2005), the isotype frequencies of cultivars were different from those of the wild populations studied in France (Bonnefoi, 1984), Italy (Villani et al., 1991a), and Turkey (Villani et al., 1991b). The observed heterozygosity $\left(\mathrm{H}_{\mathrm{o}}\right)$ was higher than the expected heterozygosity $\left(\mathrm{H}_{\mathrm{t}}\right)$ as was found previously (Pereira-Lorenzo et al., 1996b). The inbreeding coefficient $\left(\mathrm{F}_{\text {is }}\right)$ in Galicia showed a significant $(P<0.05)$ departure from HardyWeinberg proportions, with an excess of heterozygotes at each locus. In contrast, Mdh3 and Pgi loci in Asturias did not show significantly departure from Hardy-Weinberg expectations. The average multilocus inbreeding coefficient $\left(\mathrm{F}_{\text {is }}\right)$ for all samples was -0.132 , a significant $(P<0.001)$ overall departure from Hardy-Weinberg proportions. 
Table 1. Chestnut accessions and cultivars from five regions of Spain studied by morphology and isoenzymes.

\begin{tabular}{|c|c|c|c|c|c|c|c|c|}
\hline $\begin{array}{l}\text { Accessions } \\
\text { (no.) }\end{array}$ & Cultivar & Region & $\begin{array}{l}\text { Accessions } \\
\text { (no.) }\end{array}$ & Cultivar & Region & $\begin{array}{l}\text { Accessions } \\
\text { (no.) }\end{array}$ & Cultivar & Region \\
\hline 1 & Capilla & Andalucía & 7 & Pelona & Asturias & 2 & Galega & Galicia \\
\hline 2 & Comisaria & Andalucía & 2 & Pelosa & Asturias & 7 & Garrida & Galicia \\
\hline 2 & Corriente & Andalucía & 4 & Ramiega & Asturias & 1 & Garriga & Galicia \\
\hline 2 & Dieguina & Andalucía & 15 & Rapuca & Asturias & 2 & Horrea & Galicia \\
\hline 2 & Helechal & Andalucía & 1 & Ravexa & Asturias & 10 & Inxerta & Galicia \\
\hline 4 & Pelona & Andalucía & 3 & Roxa & Asturias & 3 & Lebre & Galicia \\
\hline 6 & Pilonga & Andalucía & 1 & Roxina & Asturias & 4 & Lemés & Galicia \\
\hline 4 & Planta Alajar & Andalucía & 2 & Rubia & Asturias & 8 & Longal & Galicia \\
\hline 1 & Rubia & Andalucía & 1 & Seronda & Asturias & 12 & Loura & Galicia \\
\hline 3 & Temprana & Andalucía & 4 & Sevillana & Asturias & 11 & Luguesa & Galicia \\
\hline 2 & Tomasa & Andalucía & 1 & Tamón & Asturias & 1 & Mazaíño & Galicia \\
\hline 2 & Vázquez & Andalucía & 1 & Tixera & Asturias & 1 & Migueliña & Galicia \\
\hline 2 & Argua & Asturias & 29 & Valduna & Asturias & 1 & Monfortina & Galicia \\
\hline 2 & Baragaña & Asturias & 8 & Vaquera & Asturias & 13 & Negral & Galicia \\
\hline 1 & Boroñona & Asturias & 4 & Vegamesada & Asturias & 3 & Ouriza & Galicia \\
\hline 1 & Brullina & Asturias & 15 & Verde & Asturias & 2 & Outeira & Galicia \\
\hline 2 & Calva & Asturias & 2 & Villaviciosa & Asturias & 2 & País & Galicia \\
\hline 1 & Cañuela & Asturias & 2 & Vizcaína & Asturias & 1 & Pallarega & Galicia \\
\hline 1 & Caranquexa & Asturias & 2 & Xidra & Asturias & 1 & Paradesa & Galicia \\
\hline 1 & Carreña & Asturias & 1 & Xímara & Asturias & 16 & Parede & Galicia \\
\hline 1 & Cerbana & Asturias & 5 & Zapatona & Asturias & 1 & Patacuda & Galicia \\
\hline 15 & Chamberga & Asturias & 1 & Brava & Castilla-León & 5 & Pelado & Galicia \\
\hline 1 & Chancloia & Asturias & 2 & Gallego & Castilla-León & 1 & Peluda & Galicia \\
\hline 1 & Cofina & Asturias & 6 & Injerto, Injerta & Castilla-León & 2 & Picona & Galicia \\
\hline 1 & Colunga & Asturias & 3 & Marelo & Castilla-León & 11 & Ponteareas & Galicia \\
\hline 4 & Crespa & Asturias & 8 & Negral & Castilla-León & 2 & Porteliña & Galicia \\
\hline 1 & Cruz & Asturias & 2 & Parede & Castilla-León & 2 & Portugués & Galicia \\
\hline 10 & Doriga & Asturias & 1 & Rapado & Castilla-León & 4 & Pozoredondo & Galicia \\
\hline 1 & Escamplero & Asturias & 1 & Rapega & Castilla-León & 4 & Praga d'Afora & Galicia \\
\hline 1 & Fano & Asturias & 1 & Verdello & Castilla-León & 9 & Presa & Galicia \\
\hline 2 & Feltrona & Asturias & 1 & Injerta & Extremadura & 1 & Puga & Galicia \\
\hline 8 & Forniega & Asturias & 3 & Verata & Extremadura & 5 & Puga do Bolo & Galicia \\
\hline 14 & Galega & Asturias & 3 & Abadá & Galicia & 1 & Puga do Receiro & Galicia \\
\hline 5 & Grúa & Asturias & 1 & Abarcá & Galicia & 16 & Raigona & Galicia \\
\hline 8 & Injerta & Asturias & 25 & Amarelante & Galicia & 1 & Rañuda & Galicia \\
\hline 3 & Largaña & Asturias & 1 & Anaxa & Galicia & 7 & Rapada & Galicia \\
\hline 3 & Leinova & Asturias & 1 & Anchas & Galicia & 1 & Redondo & Galicia \\
\hline 9 & Llanisca & Asturias & 2 & Arial & Galicia & 2 & Riá & Galicia \\
\hline 1 & Loura & Asturias & 5 & Bermella & Galicia & 1 & Ribeirá & Galicia \\
\hline 1 & Mariana & Asturias & 16 & Blanca & Galicia & 1 & Rosenda & Galicia \\
\hline 2 & Marimoeche & Asturias & 1 & Bolesa & Galicia & 1 & Rozada & Galicia \\
\hline 2 & Marina & Asturias & 5 & Bravoleirado & Galicia & 5 & Salnesa & Galicia \\
\hline 1 & Mediana & Asturias & 3 & Cabezuda & Galicia & 2 & Sergude & Galicia \\
\hline 4 & Miguelina & Asturias & 4 & Calva & Galicia & 2 & Serodia & Galicia \\
\hline 1 & Montesín & Asturias & 3 & Campilla & Galicia & 1 & Soutogrande & Galicia \\
\hline 2 & Mourisco & Asturias & 1 & Carrelao & Galicia & 1 & Tarabelao & Galicia \\
\hline 10 & Nargana & Asturias & 1 & Castellana & Galicia & 9 & Temperá & Galicia \\
\hline 4 & Navexa & Asturias & 1 & Caurelal & Galicia & 2 & Torbeana & Galicia \\
\hline 3 & Negral & Asturias & 2 & Cedo & Galicia & 1 & Toubesa & Galicia \\
\hline 7 & Ordaliega & Asturias & 3 & Cerdedo & Galicia & 1 & Veiguiña & Galicia \\
\hline 4 & Padana, Pagana & Asturias & 2 & Cerreda & Galicia & 8 & Ventura & Galicia \\
\hline 1 & Palaciana & Asturias & 7 & Courelá & Galicia & 17 & Verde & Galicia \\
\hline 1 & Panchina & Asturias & 3 & Culona & Galicia & 2 & Vileta & Galicia \\
\hline 33 & Parede & Asturias & 1 & Curuxa & Galicia & 1 & Villarenga & Galicia \\
\hline 6 & Parruca & Asturias & 2 & Desgrañadiza & Galicia & 7 & Xabrega & Galicia \\
\hline \multirow[t]{2}{*}{1} & Pelgo & Asturias & 16 & Famosa & Galicia & 1 & Xilimendra & Galicia \\
\hline & & & & & & 701 & Total & \\
\hline
\end{tabular}


Table 2. Estimates of genetic diversity parameters in 611 accessions corresponding to 163 chestnut cultivars located in five regions of Spain: Asturias, Galicia, and Castilla-León in northern Spain; Extremadura in central Spain; and Andalucía in southern Spain.

\begin{tabular}{|c|c|c|c|c|c|c|c|}
\hline \multirow[b]{3}{*}{ Enzyme } & \multirow[b]{3}{*}{ Allele } & \multicolumn{6}{|c|}{ Allele frequencies } \\
\hline & & Spain & Asturias & Galicia & Andalucía & Castilla-León & Extremadura \\
\hline & & $\mathrm{N}=611^{\mathrm{z}}$ & $\mathrm{N}=258$ & $\mathrm{~N}=300$ & $\mathrm{~N}=28$ & $\mathrm{~N}=21$ & $\mathrm{~N}=4$ \\
\hline$\overline{\text { Mdh1 }}$ & $M d h 1-b$ & 1.000 & 1.000 & 1.000 & 1.000 & 1.000 & 1.000 \\
\hline \multirow[t]{2}{*}{ Mdh2 } & $M d h 2-a$ & 0.736 & 0.653 & 0.807 & 0.768 & 0.762 & 0.500 \\
\hline & $M d h 2-b$ & 0.264 & 0.347 & 0.193 & 0.232 & 0.238 & 0.500 \\
\hline \multirow[t]{2}{*}{ Mdh3 } & $M d h 3-a$ & 0.159 & 0.091 & 0.217 & 0.125 & 0.238 & 0.000 \\
\hline & $M d h 3-b$ & 0.841 & 0.909 & 0.783 & 0.875 & 0.762 & 1.000 \\
\hline \multirow[t]{3}{*}{ Skdh } & $S k d h-a$ & 0.420 & 0.432 & 0.435 & 0.179 & 0.310 & 0.750 \\
\hline & $S k d h-b$ & 0.090 & 0.091 & 0.097 & 0.071 & 0.024 & 0.000 \\
\hline & $S k d h-c$ & 0.490 & 0.477 & 0.468 & 0.750 & 0.667 & 0.250 \\
\hline \multirow[t]{2}{*}{ Idh } & $I d h-a$ & 0.920 & 0.996 & 0.843 & 0.964 & 1.000 & 1.000 \\
\hline & $I d h-b$ & 0.080 & 0.004 & 0.157 & 0.036 & 0.000 & 0.000 \\
\hline \multirow[t]{3}{*}{ Pgi } & $P g i-a$ & 0.608 & 0.690 & 0.525 & 0.768 & 0.619 & 0.375 \\
\hline & $P g i-b$ & 0.031 & 0.035 & 0.030 & 0.000 & 0.048 & 0.000 \\
\hline & $P g i-c$ & 0.361 & 0.275 & 0.445 & 0.232 & 0.333 & 0.625 \\
\hline Enzyme & & \multicolumn{6}{|c|}{$\mathrm{F}_{\mathrm{is}}{ }^{\mathrm{y}}$} \\
\hline$\overline{M d h 2}$ & & $-0.222^{* * * *}$ & $-0.204^{* * *}$ & $-0.217^{* * * * *}$ & -0.286 & -0.290 & -1.000 \\
\hline Mdh3 & & $-0.209^{* * * *}$ & -0.051 & $-0.275^{* * * *}$ & -0.125 & -0.290 & $\mathrm{NA}^{\mathrm{s}}$ \\
\hline Skdh & & $-0.152^{* * * *}$ & $-0.186^{* * * * *}$ & $-0.150^{* * * * *}$ & 0.303 & -0.013 & -0.200 \\
\hline Idh & & $-0.174^{* * * *}$ & $1.000^{* * * * *}$ & $-0.184^{* * *}$ & -0.019 & NA & NA \\
\hline Pgi & & $0.016^{* * *}$ & -0.032 & $0.082^{* * * * *}$ & -0.286 & -0.111 & -0.500 \\
\hline Average & & $-0.132^{* * * * *}$ & & & & & \\
\hline Enzyme & & $\mathrm{H}_{\mathrm{o}}{ }^{\mathrm{x}}$ & $\mathrm{H}_{\mathrm{s}}{ }^{\mathrm{w}}$ & $\mathrm{H}_{\mathrm{t}}^{\mathrm{v}}$ & $\mathrm{F}_{\text {is }}$ & $\mathrm{F}_{\mathrm{it}}^{\mathrm{u}}$ & $\mathrm{F}_{\mathrm{st}} \mathrm{t}$ \\
\hline Mdh2 & & 0.573 & 0.405 & 0.423 & -0.222 & -0.165 & 0.046 \\
\hline Mdh3 & & 0.267 & 0.223 & 0.234 & -0.209 & -0.152 & 0.047 \\
\hline Skdh & & 0.525 & 0.495 & 0.550 & -0.152 & -0.126 & 0.022 \\
\hline Idh & & 0.079 & 0.070 & 0.076 & -0.174 & -0.030 & 0.122 \\
\hline Pgi & & 0.546 & 0.474 & 0.502 & 0.016 & 0.063 & 0.048 \\
\hline Average & & 0.398 & 0.333 & 0.357 & -0.132 & -0.080 & 0.046 \\
\hline
\end{tabular}

${ }^{2} \mathrm{~N}=$ number of accessions.

$\mathrm{y}_{\mathrm{is}}=$ deviations from Hardy-Weinberg equilibrium among individuals within populations.

${ }^{\times} \mathrm{H}_{\mathrm{o}}=$ observed heterozygosities.

${ }^{\mathrm{w}} \mathrm{H}_{\mathrm{s}}=$ expected heterozygosities within populations.

${ }^{v} \mathrm{H}_{\mathrm{t}}=$ total diversity.

$\mathrm{uF}_{\mathrm{it}}=$ total deviations from Hardy-Weinberg among individuals.

${ }^{\mathrm{t}} \mathrm{F}_{\mathrm{st}}=$ deviation from Hardy-Weinberg among populations.

${ }^{\mathrm{N}} \mathrm{NA}=$ missing data.

Nut SIZE. In Spain the average NNK was 110 nuts/kg (Table 3 ), but with variation ranging from $38-343$ nuts $/ \mathrm{kg}$. There are also regional differences in NNK; with 119 nuts/kg in Asturias (smallest nuts), 113 nuts/kg in Castilla-León (El Bierzo), 108 nuts $/ \mathrm{kg}$ in Galicia, 87 in Extremadura (Guadalupe) and, finally, 73 in Andalucía. Nuts from Andalucía weighed on average 14-17 $\mathrm{g}$, the highest in Spain. Classifying all the accessions from Spain showed that $58 \%$ produced more than 100 nuts $/ \mathrm{kg}$; these accessions have a low market value. Only $7 \%$ of the accessions produced nuts averaging more than $15 \mathrm{~g}$, and these were primarily from Andalucía. Chestnuts from Asturias were similar to those from Galicia in WLN, with only $2 \%$ to $6 \%$ of the accessions producing nuts over $15 \mathrm{~g}$.

Harvesting time. Early nut ripening is a useful genetic character for breeding. In Spain, harvest occurs from around 15 Sept. until mid-November. Only $16 \%$ of the accessions from Andalucía in southern Spain produced VEA nuts. In Asturias and Castilla-León, only between 2\% to 5\% of accessions produced EAR nuts, while in Galicia, late production is more common $(43 \%)$.

Nut SHAPE. The most frequent shapes in Spain were the ellipti- cal-short forms $(110<\mathrm{WRL}<120)$ found in $46 \%$ of accessions, the elliptical-triangular form $(100<W R L<110)$ in $30 \%$ of accessions, and the elliptical-broad (WRL $>120$ ) in $18 \%$ of accessions (classification according to Pereira-Lorenzo and Ramos-Cabrer, 2004). Triangular-shaped nuts (WRL $<100)$ were the least frequent category, and found in only $6 \%$ of accessions. The elliptical-short shape is predominant in northern Spain (Asturias, Castilla-León, and Asturias) while in the south, elliptical-triangular forms are more frequent.

CATKIN TYPE. In Spain, CLO is predominant (43\% of the total accessions, $66 \%$ in Asturias, and $84 \%$ in Andalucía). In the high rainfall Galicia, CME is most frequent. Castilla-León appears to be a transition region between the CME of Galicia and the CLO of Asturias. Only CLO produces abundant pollen.

SPINE LENGTH. The length of the spines on burs is another character to distinguishing cultivars. BTS make harvesting (normally by hand) easier. In Galicia and Asturias, 19\% and 11\% of cultivars respectively had burs with BTS. In Andalucía, BTL were the most frequent (84\%).

PDN. Non-divided nuts in C. sativa are thought to have resulted from selection (Breviglieri, 1955). In Spain, only 12\% of 


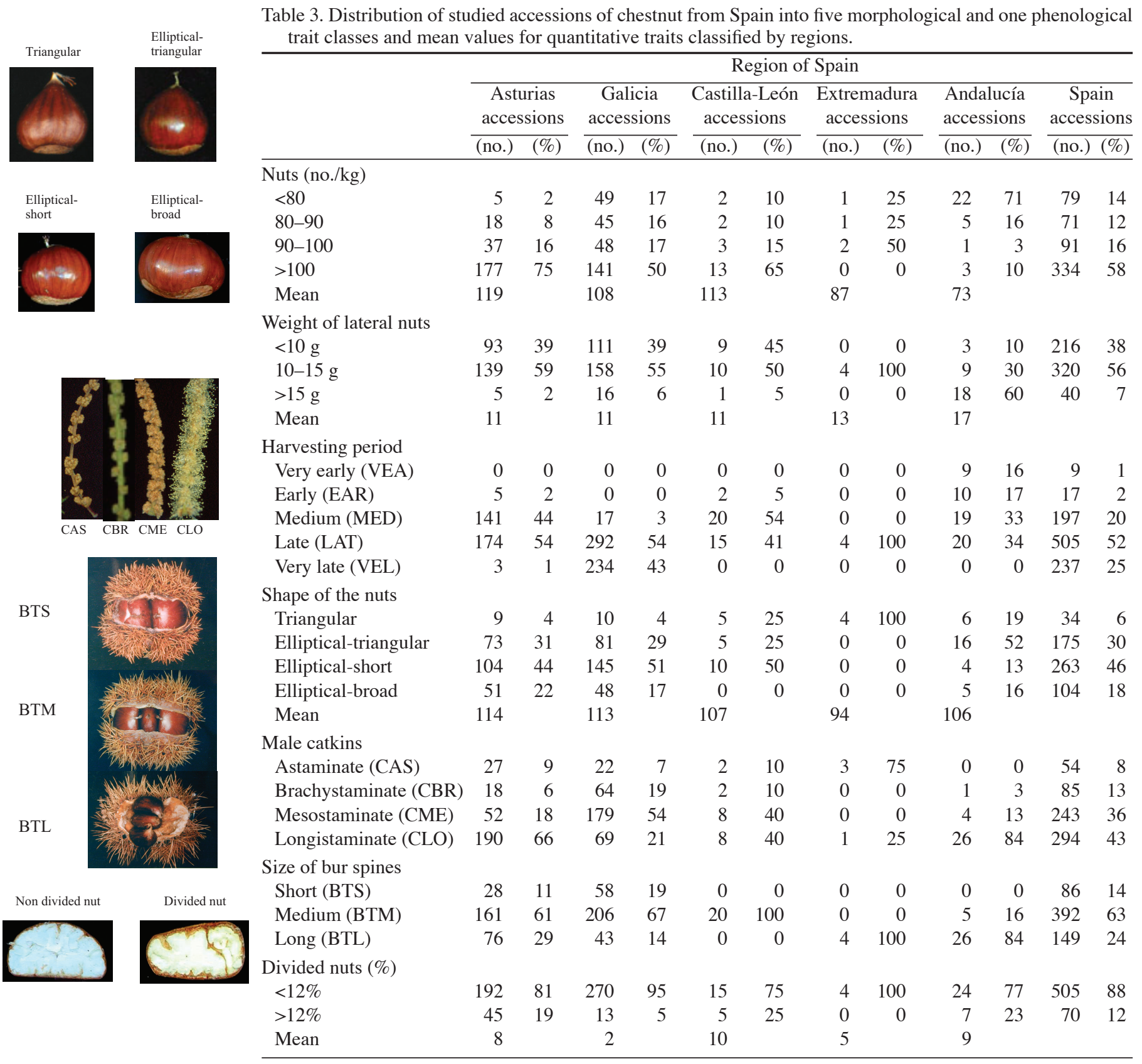

the accessions produced more than $12 \%$ of PDN. Higher proportion were found in Andalucía and Castilla-León (23\% and 25\%), while in Galicia and Extremadura less than 5\% of the accessions produced more than $12 \%$ of PDN.

Classification by isozymes, morphology and Phenology. Using PCA we reduced 27 variables ( 15 corresponding to the six morphological traits and 12 to isotypes) to $11 \mathrm{PCs}$ (PC1 to PC11) with an eigen value over 1 . Those 11 PCs accounted for $78 \%$ of the total variance. Characteristics with highest eigen vectors were as follows: PC1, Mdh2100, Mdh2110, Idh100, Idh105, CME and CLO and LAT; PC2, Mdh395, Mdh3100, Pgi90 and Pgi100; PC3, Skdh100; PC4, BTM and BTL, VEA and EAR and NNK; PC5, BTM and BTL; PC6, Skdh97, CAS and NNK; PC7, BTS and BTM; PC8, Skdh97 and Skdh100 and EAR; PC9, Skdh97, WLR and BTS; PC10, CBR; and PC11, Pgi92 and CAS. The UPGMA cluster analysis carried out on 501 accessions produced 77 clusters when the dendogram is cut at a Mahalanobis distance of 0.6 (Fig. 2), 49 of them are shown in Table 4. Morphology confirmed most of the clusters with isozymes. Only two clusters were differentiated on morphological characteristics (WLR for cluster 12 and male catkins for cluster 24).

Possible synonymies were identified in Fig. 2 and Table 4 (synonyms are in parentheses): 1) cluster 1 - 'Tempera' and 'Cabezuda'; 2) cluster 7-'Amarelante', 'Abarcá', 'Anchas', and 'Desgrañadiza'; 3) cluster 7- 'Inxerta' and 'Porteliña'; 4) cluster 10-'Luguesa' and 'Tarabelao'; 5) cluster 10-'Presa' and 'Veiguiña'; 6) cluster 12 - 'Injerta' and 'Gallego'; 7) cluster 12-'Parede', 'Padana', 'Paradesa', and 'Xidra'; 8) cluster $13-$ 'Chamberga' and 'Valduna'; 9) cluster 14- 'Negral', 'Courelá', 'Riá', 'Vileta', and 'Xabrega'; 10) cluster 15-'Negral' and 'Vaquera'; 11) cluster 20 - 'Galega', 'Colunga', and 'Zapatona'; 12) cluster 20-'Largana', 'Nargana', and 'Parruca'; 13) cluster $22-$ 'Pelona' and 'Feltrona'; and 14) cluster 50-'Negral' and 'Blanca'. 


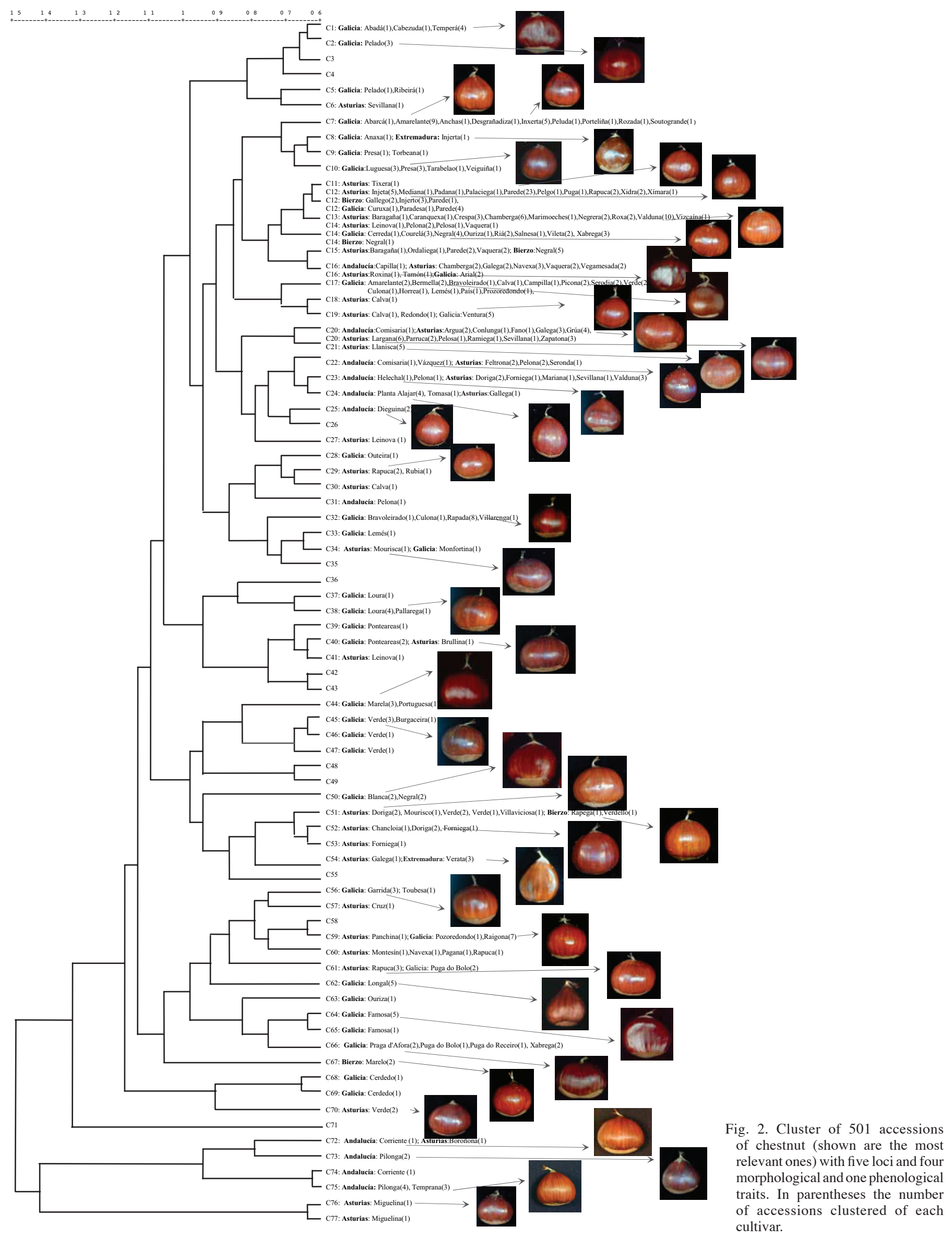


Table 4. Classification of Spanish chestnut cultivars and synonyms from five regions of Spain using five morphological and one phenological traits and genotypes described with four isoenzymes.

\begin{tabular}{|c|c|c|c|c|c|c|c|c|c|c|c|c|c|c|c|}
\hline $\begin{array}{l}\text { Cultivars (synonyms in } \\
\text { parentheses) }\end{array}$ & Region & $\begin{array}{c}\text { Accessions } \\
\text { (no.) }\end{array}$ & Genotype & Cluster & Mdh2 & Mdh3 & Skdh & Idh & Pgi & $\begin{array}{c}\text { Nuts } \\
\text { (no./kg) }\end{array}$ & $\begin{array}{c}\text { Harvesting } \mathrm{N} \\
\text { period }^{2}\end{array}$ & Nut shape ${ }^{y}$ & $\begin{array}{l}\text { Type } \\
\text { of } \\
\text { catkin }^{\mathrm{x}}\end{array}$ & $\begin{array}{l}\text { Spine } \\
\text { length }^{w}\end{array}$ & $\begin{array}{l}\mathrm{PDN}^{\mathrm{v}} \\
(\%)\end{array}$ \\
\hline Temperá (Cabezuda) & Galicia & 5 & 1 & 1 & $\mathrm{ab}$ & $\mathrm{bb}$ & $\mathrm{bc}$ & aa & aa & 83 & LAT & Ell-Short & CME & BTM & 2 \\
\hline Corriente & Andalucía & 1 & 1 & 72 & $\mathrm{ab}$ & $\mathrm{bb}$ & $\mathrm{bc}$ & aa & aа & 70 & VEA & Ell-Short & CLO & BTM & 13 \\
\hline Pelado & Galicia & 3 & 2 & 2 & aa & $\mathrm{ab}$ & $\mathrm{bc}$ & aa & aa & 88 & LAT,VEL $\mathrm{E}$ & Ell-Broad & CME & BTM & 2 \\
\hline Marela & Galicia & 3 & 2 & 44 & aa & $a b$ & $\mathrm{bc}$ & aa & aa & 131 & LAT,VEL I & Ell-Short & CAS & BTM & 7 \\
\hline Amarelante (Abarcá, Anchas, & Galicia & 12 & 3 & 7 & $\mathrm{ab}$ & $\mathrm{ab}$ & ac & aa & $\mathrm{cc}$ & 88 & LAT,VEL & Ell-Short & CME & BTM & 1 \\
\hline \multicolumn{16}{|l|}{ Desgrañadiza) } \\
\hline Inxerta (Porteliña) & Galicia & 5 & 4 & 7 & aa & $a b$ & $\mathrm{bc}$ & aa & $\mathrm{cc}$ & 76 & LAT,VEL & Ell-Tr & CME & BTM & 0 \\
\hline Injerta & Extremadura & 1 & 5 & 8 & $\mathrm{ab}$ & $\mathrm{bb}$ & $\mathrm{ac}$ & aa & $\mathrm{cc}$ & 78 & LAT & Triang & CLO & BTL & 0 \\
\hline Luguesa (Tarabelao) & Galicia & 4 & 6 & 10 & aa & $\mathrm{bb}$ & $\mathrm{ab}$ & aa & $\mathrm{cc}$ & 97 & LAT,VEL I & Ell-Short & CME & BTM & 1 \\
\hline Presa (Veiguiña) & Galicia & 4 & 7 & 10 & aa & $\mathrm{bb}$ & $\mathrm{cc}$ & aa & $\mathrm{ac}$ & 97 & LAT,VEL I & Ell-Short & CME & BTM & 1 \\
\hline Galega & Asturias & 2 & 7 & 16 & aa & $\mathrm{bb}$ & $\mathrm{cc}$ & aa & $\mathrm{ac}$ & 130 & MED & Ell-Tr & CLO & BTM & 5 \\
\hline Injerta & Asturias & 5 & 8 & 12 & aa & $\mathrm{bb}$ & ac & aa & aa & 152 & MED,LAT & Ell-Tr & CLO & BTM & 13 \\
\hline Injerta (Gallego) & Castilla-León & 5 & 8 & 12 & aa & $\mathrm{bb}$ & $\mathrm{ac}$ & aa & aа & 141 & MED,LAT & Triang & CME & BTM & 7 \\
\hline Parede (Padana, Paradesa, & Asturias, & 34 & 8 & 12 & aa & $\mathrm{bb}$ & ac & aa & aa & 135 & MED,LAT, & Ell-Tr & CME & BTM & 6 \\
\hline \multirow[t]{2}{*}{ Xidra) } & Castilla-León, & & & & & & & & & & VEL & & & & \\
\hline & Galicia & & & & & & & & & & & & & & \\
\hline Rapuca & Asturias & 3 & 8 & 61 & aa & $\mathrm{bb}$ & $\mathrm{ac}$ & aa & aа & 125 & MED & Ell-Short & CBR & BTS & 3 \\
\hline Chamberga (Valduna) & Asturias & 16 & 9 & 13 & $\mathrm{ab}$ & $\mathrm{bb}$ & $\mathrm{ac}$ & aa & aа & 96 & MED,LAT $\mathrm{E}$ & Ell-Broad & CLO & BTM & 2 \\
\hline Valduna2 & Asturias & 3 & 9 & 23 & $\mathrm{ab}$ & $\mathrm{bb}$ & $\mathrm{ac}$ & aa & aa & 99 & MED,LAT I & Ell-Short & CLO & BTL & 4 \\
\hline Crespa & Asturias & 3 & 10 & 13 & $\mathrm{bb}$ & $\mathrm{bb}$ & aa & aa & $\mathrm{ac}$ & 164 & MED,LAT & Ell-Short & CLO & BTM & 4 \\
\hline Negrera & Asturias & 2 & 11 & 13 & aa & $\mathrm{bb}$ & aa & aa & aa & 115 & MED,LAT & Ell-Short & CLO & BTM & 4 \\
\hline Roxa & Asturias & 2 & 12 & 13 & $\mathrm{ab}$ & $\mathrm{bb}$ & aa & aа & $\mathrm{cc}$ & 135 & MED,LAT & Ell-Short & CLO & BTM & 12 \\
\hline Vizcaína & Asturias & 2 & 13 & 13 & $\mathrm{ab}$ & $\mathrm{bb}$ & aa & aa & aa & 111 & MED,LAT & Ell-Tr & CLO & BTM & 7 \\
\hline Grúa & Asturias & 4 & 13 & 20 & $\mathrm{ab}$ & $\mathrm{bb}$ & aa & aa & aa & 90 & MED,VEL & Ell-Broad & CLO & BTL & 7 \\
\hline Garrida & Galicia & 3 & 13 & 56 & $\mathrm{ab}$ & $\mathrm{bb}$ & aa & aa & aa & 74 & LAT,VEL & Ell-Short & $\mathrm{CBR}$ & BTM & 2 \\
\hline Negral (Courelá, Riá, Vileta, & Galicia, & 15 & 14 & 14 & $\mathrm{ab}$ & $\mathrm{ab}$ & aa & aa & ac & 103 & EAR,MED, & Ell-Short & CLO & BTM & 2 \\
\hline Xabrega) & Castilla-León & & & & & & & & & & LAT & & & & \\
\hline Pelona & Asturias & 2 & 15 & 14 & $\mathrm{ab}$ & $a b$ & $\mathrm{ac}$ & aa & aa & 173 & MED,LAT & Ell-Tr & CLO & BTM & 3 \\
\hline Pelona (Feltrona) & Asturias & 4 & 15 & 22 & $\mathrm{ab}$ & $\mathrm{ab}$ & $\mathrm{ac}$ & aa & aa & 117 & MED,LAT & Ell-Short & CLO & BTL & 1 \\
\hline \multirow[t]{2}{*}{ Negral (Vaquera) } & Castilla-León, & 5 & 16 & 15 & $\mathrm{ab}$ & $\mathrm{ab}$ & $\mathrm{cc}$ & aa & ac & 98 & MED,LAT & Ell-Short & CLO & BTM & 7 \\
\hline & Asturias & & & & & & & & & & & & & & \\
\hline Arial & Galicia & 2 & 17 & 16 & aa & $\mathrm{bb}$ & $\mathrm{cc}$ & aa & aa & 81 & LAT,VEL & Ell-Tr & CLO & BTM & 2 \\
\hline Navexa & Asturias & 3 & 17 & 16 & aa & $\mathrm{bb}$ & $\mathrm{cc}$ & aa & aа & 104 & LAT & Ell-Short & CLO & BTM & 6 \\
\hline Vegamesada & Asturias & 2 & 17 & 16 & aa & $\mathrm{bb}$ & $\mathrm{cc}$ & aa & aa & 92 & MED,LAT & Ell-Short & $\mathrm{CME}$ & BTM & 6 \\
\hline Planta Alajar & Andalucía & 4 & 17 & 24 & aa & $\mathrm{bb}$ & $\mathrm{cc}$ & aa & aa & 71 & MED,LAT & Triang & CME & BTL & 11 \\
\hline
\end{tabular}

Table 4. Continued on next page. 
Table 4. Continued from previous page.

\begin{tabular}{|c|c|c|c|c|c|c|c|c|c|c|c|c|c|c|c|}
\hline $\begin{array}{l}\text { Cultivars (synonyms in } \\
\text { parentheses) }\end{array}$ & Region & $\begin{array}{c}\text { Accessions } \\
\text { (no.) }\end{array}$ & Genotype & Cluster & Mdh2 & Mdh3 & Skdh & Idh & Pgi & $\begin{array}{c}\text { Nuts } \\
\text { (no./kg) }\end{array}$ & $\begin{array}{c}\text { Harvesting } \\
\text { period }^{\mathrm{z}}\end{array}$ & Nut shape ${ }^{y}$ & $\begin{array}{c}\text { Type } \\
\text { of } \\
\text { catkin }\end{array}$ & $\begin{array}{l}\text { Spine } \\
\text { length }\end{array}$ & $\begin{array}{r}\text { PDN } \\
(\%)\end{array}$ \\
\hline Pilonga & Andalucía & 2 & 17 & 73 & aa & $\mathrm{bb}$ & $\mathrm{cc}$ & aa & aa & 68 & $\begin{array}{r}\text { EAR,ME } \\
\text { D,LAT }\end{array}$ & Ell-Tr & CLO & BTL & 9 \\
\hline Corriente & Andalucía & 1 & 17 & 74 & aa & $\mathrm{bb}$ & $\mathrm{cc}$ & aa & aa & 58 & VEA,EAR & Ell-Broad & CLO & BTM & 20 \\
\hline Pilonga & Andalucía & 4 & 17 & 75 & aa & $\mathrm{bb}$ & $\mathrm{cc}$ & aa & aa & 63 & VEA,EAR & Ell-Tr & CLO & BTL & 8 \\
\hline Bermella & Galicia & 2 & 18 & 17 & $a b$ & $\mathrm{bb}$ & $\mathrm{ac}$ & $\mathrm{ab}$ & $\mathrm{ac}$ & 145 & LAT,VEL & Ell-Short & $\mathrm{CME}$ & BTM & 11 \\
\hline Picona & Galicia & 2 & 19 & 17 & aa & $\mathrm{bb}$ & $\mathrm{ac}$ & $\mathrm{ab}$ & aa & 119 & LAT,VEL & Ell-Short & $\begin{array}{l}\text { CME, } \\
\text { CLO }\end{array}$ & BTM & 14 \\
\hline Serodia & Galicia & 2 & 19 & 17 & aa & $\mathrm{bb}$ & $\mathrm{ac}$ & $\mathrm{ab}$ & aa & 101 & LAT,VEL & Ell-Broad & CLO & BTM & 1 \\
\hline Rapada & Galicia & 8 & 19 & 32 & aa & $\mathrm{bb}$ & $\mathrm{ac}$ & $\mathrm{ab}$ & aa & 103 & LAT,VEL & Ell-Short & $\mathrm{CME}$ & BTS & 4 \\
\hline Praga Dafora & Galicia & 2 & 19 & 66 & aa & $\mathrm{bb}$ & $\mathrm{ac}$ & $\mathrm{ab}$ & aa & 98 & LAT,VEL & Ell-Short & CBR & BTM & 1 \\
\hline Ventura & Galicia & 5 & 20 & 19 & aa & $\mathrm{ab}$ & $\mathrm{bc}$ & $\mathrm{ab}$ & aa & 90 & LAT & Ell-Tr & $\mathrm{CME}$ & BTM & 0 \\
\hline Argua & Asturias & 2 & 21 & 20 & aa & $\mathrm{bb}$ & $\mathrm{ab}$ & aa & $\mathrm{ac}$ & 95 & LAT,VEL & Ell-Broad & CLO & BTL & 5 \\
\hline Galega (Colunga, Zapatona) & Asturias & 7 & 22 & 20 & $a b$ & $\mathrm{bb}$ & $a b$ & aa & aa & 93 & MED,LAT & Ell-Short & CLO & BTL & 8 \\
\hline Boroñona & Asturias & 1 & 22 & 72 & $\mathrm{ab}$ & $\mathrm{bb}$ & $\mathrm{ab}$ & aa & aa & 111 & LAT & Ell-Short & CLO & BTM & 4 \\
\hline Largaña (Nargana, Parruca) & Asturias & 8 & 23 & 20 & $\mathrm{ab}$ & $\mathrm{bb}$ & aa & aa & ac & 123 & MED,LAT & Ell-Short & CLO & BTL & 5 \\
\hline Verata & Extremadura & 3 & 23 & 54 & $\mathrm{ab}$ & $\mathrm{bb}$ & aa & aa & ac & 90 & LAT & Triang & CAS & BTL & 6 \\
\hline Llanisca & Asturias & 4 & 24 & 21 & $\mathrm{bb}$ & $\mathrm{bb}$ & $\mathrm{ab}$ & aa & $\mathrm{cc}$ & 123 & LAT & Ell-Broad & $\mathrm{CLO}$ & BTL & 10 \\
\hline Comisaria & Andalucía & 1 & 25 & 22 & aa & $\mathrm{ab}$ & $\mathrm{cc}$ & aa & ac & 69 & LAT & Ell-Tr & CLO & BTL & 4 \\
\hline Pelona & Andalucía & 1 & 26 & 22 & aa & $\mathrm{ab}$ & $\mathrm{ac}$ & aa & $\mathrm{ac}$ & 72 & MED,LAT & Ell-Tr & CLO & BTL & 8 \\
\hline Vázquez & Andalucía & 1 & 27 & 22 & $\mathrm{ab}$ & $\mathrm{ab}$ & $\mathrm{bc}$ & aa & $\mathrm{ac}$ & 68 & MED,LAT & Ell-Short & $\mathrm{CLO}$ & BTL & 0 \\
\hline Rapuca & Asturias & 2 & 28 & 29 & aa & $\mathrm{ab}$ & $\mathrm{ac}$ & aa & ac & 134 & MED,LAT & Ell-Short & $\mathrm{CME}$ & BTS & 6 \\
\hline Verde & Galicia & 3 & 28 & 45 & aa & $\mathrm{ab}$ & $\mathrm{ac}$ & aa & $\mathrm{ac}$ & 105 & VEL & Ell-Broad & CAS & BTM & 3 \\
\hline Verde & Galicia & 1 & 28 & 47 & aa & $\mathrm{ab}$ & $\mathrm{ac}$ & aa & $\mathrm{ac}$ & 97 & VEL & Ell-Broad & CAS & BTS & 1 \\
\hline Doriga & Asturias & 2 & 29 & 23 & $\mathrm{ab}$ & $\mathrm{bb}$ & $\mathrm{cc}$ & aа & ac & 154 & MED & Ell-Short & CLO & BTL & 8 \\
\hline Doriga & Asturias & 2 & 29 & 51 & $\mathrm{ab}$ & $\mathrm{bb}$ & $\mathrm{cc}$ & aa & $\mathrm{ac}$ & 134 & MED,LAT & Ell-Short & CAS & BTM & 13 \\
\hline Verde & Asturias & 2 & 29 & 51 & $\mathrm{ab}$ & $\mathrm{bb}$ & $\mathrm{cc}$ & aa & $\mathrm{ac}$ & 147 & MED,LAT & Ell-Short & CAS & BTM & 15 \\
\hline Doriga & Asturias & 2 & 29 & 52 & $\mathrm{ab}$ & $\mathrm{bb}$ & $\mathrm{cc}$ & aa & ac & 141 & MED,LAT & Ell-Short & CAS & BTL & 9 \\
\hline Helechal & Andalucía & 1 & 30 & 23 & $\mathrm{ab}$ & $\mathrm{bb}$ & $\mathrm{ac}$ & aa & $\mathrm{ac}$ & 94 & MED,LAT & Ell-Tr & $\mathrm{CLO}$ & BTL & 8 \\
\hline Tomasa & Andalucía & 1 & 31 & 24 & $\mathrm{ab}$ & $\mathrm{bb}$ & $\mathrm{cc}$ & aa & aa & 46 & MED,LAT & Triang & CLO & BTL & 14 \\
\hline Temprana & Andalucía & 3 & 31 & 75 & $\mathrm{ab}$ & $\mathrm{bb}$ & $\mathrm{cc}$ & aa & aa & 61 & VEA,EAR & Ell-Broad & CLO & BTL & 12 \\
\hline Dieguina & Andalucía & 2 & 32 & 25 & $\mathrm{ab}$ & $\mathrm{ab}$ & $\mathrm{bc}$ & $\mathrm{ab}$ & $\mathrm{ac}$ & 87 & MED,LAT & Ell-Tr & $\begin{array}{r}\text { CME, } \\
\text { CLO }\end{array}$ & BTL & 8 \\
\hline Pelona & Andalucía & 1 & 33 & 31 & aa & $\mathrm{ab}$ & aa & aa & $\mathrm{ac}$ & 140 & MED,LAT & Triang & $\mathrm{CLO}$ & BTL & 3 \\
\hline Loura & Galicia & 1 & 34 & 37 & aa & $\mathrm{ab}$ & $\mathrm{bc}$ & aa & $\mathrm{bc}$ & 78 & LAT,VEL & Ell-Broad & CBR & BTM & 0 \\
\hline Loura & Galicia & 4 & 34 & 38 & aa & $\mathrm{ab}$ & $\mathrm{bc}$ & aa & $\mathrm{bc}$ & 82 & LAT,VEL & Ell-Broad & $\mathrm{CME}$ & BTM & 1 \\
\hline
\end{tabular}

Table 4. Continued on next page. 
Table 4. Continued from previous page.

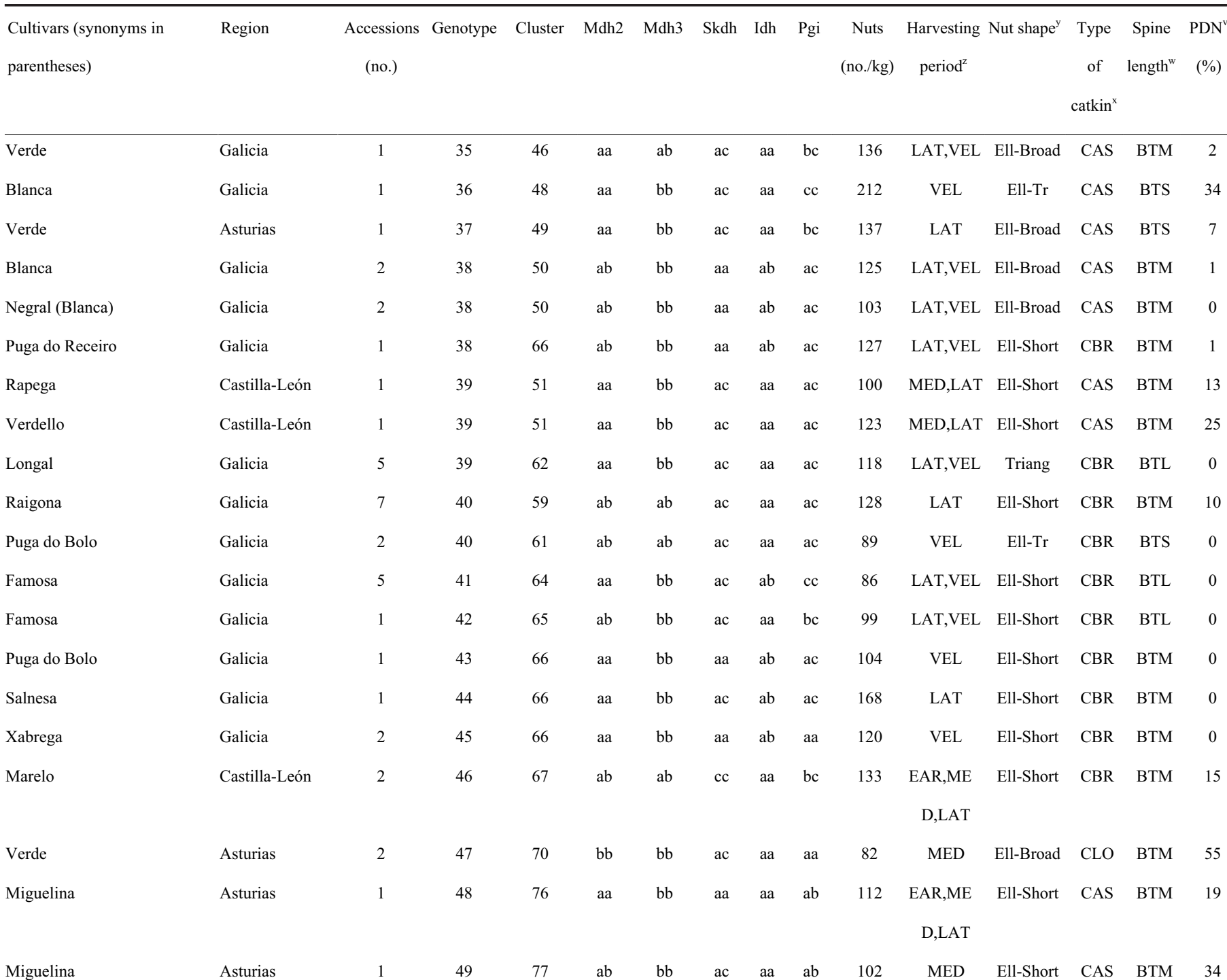

zVEA = very early, EAR = early, MED = medium, LAT = late, VEL = very late.

$y$ Triang = triangular, Ell-Tr = elliptical-triangular, Ell-Short $=$ elliptical-short, Ell-Broad = elliptical-broad .

${ }^{x} \mathrm{CAS}=$ astaminate, $\mathrm{CBR}=$ brachystaminate, $\mathrm{CME}=$ mesostaminate, $\mathrm{CLO}=$ longistaminate.

${ }^{\mathrm{w}} \mathrm{BTS}=$ short, $\mathrm{BTM}=$ medium, $\mathrm{BTL}=$ long.

$\checkmark \mathrm{PDN}=$ percentage of divided nuts.

A total of 152 Spanish cultivars were classified into 58 cultivars of major importance and 94 with minor importance, 18 of which had high intracultivar variation. A total of 14 groups of possible synonymies were detected involving 37 cultivars. We observed six groups in Galicia containing 14 cultivars, one group in CastillaLeón (El Bierzo) with two cultivars, four groups in Asturias with 10 cultivars, one group in Asturias and Castilla-León (El Bierzo) with two cultivars, and two groups in Asturias, Castilla-León (El Bierzo) and Galicia with nine cultivars.

Although 18 out of 152 cultivars classified had high intracultivar variation $(12 \%)$, the cultivars most intensively studied (three or more accessions from different localities), did not vary greatly. For instance, in Galicia and Asturias, 58\% and 57\% of accessions of a specific cultivar, respectively, were grouped in the same cluster. This suggests that a particular clone has been propagated for many years in each of these regions. 'Parede' clus- tered with 23 accessions out of 29 studied in Asturias (79\%) and coincidentally with six out of nine studied in Galicia (67\%).

\section{Discussion}

This study classified 152 Spanish chestnut cultivars into 58 of major importance and 94 of minor importance. A total of 14 groups of possible synonymies were detected involving 37 cultivars. All isotypes in this study had previously been found in Galicia (Pereira-Lorenzo et al., 1996b), suggesting that the whole population of Spanish cultivars came from a common pool. The cultivars of Galicia and Asturias can be defined as a continuum of common isotypes and cultivars and representing an ancestral gene pool. The chestnuts in Galicia (300 accessions studied by isozymes) are a good representation of the entire Spanish chestnut population (617 accessions in total) and represent a region with the highest variation in Spain. 
The excess of heterozygotes found in Galician chestnuts was similar to the studies by Stilwell et al. (2003), who used five isozymes on five natural populations of $C$. dentata in Virginia. These authors explained excess heterozygosity by the selection of heterozygous breeding material and by an absence of new recruits from other populations. These $C$. dentata populations were over 70 years old; the orchards studied here are at least 300 years old. Nevertheless, the excess of heterozygotes observed might have resulted from patterns similar to those described by Stilwell et al. (2003). Subsequent asexual propagation through grafting would maintain this situation. The F statistics indicated a small differentiation among populations $\left(\mathrm{F}_{\mathrm{st}}=0.046\right)$; this could be explained by the movement of clonal cultivars, such as 'Parede', between more than one region. Subpopulations isolated in southern Spain had lower variation.

The importance of chestnut cultivation reduces from the north to the south of Spain, as indicated by the lower number of cultivars in southern Spain. It may also be significant that there are major physical barriers between the different areas. In Andalucía the cultivars are less variable and fall into fewer groups than those in provinces of northern Spain; this could be due to the region's geographic isolation. However, cultivars from Castilla-León are grouped with the principal cultivars of Asturias and Galicia, which indicates that selection and spread in these regions were less affected by geographical location. This is reflected in little differentiation among populations $\left(\mathrm{F}_{\mathrm{st}}=0.046\right)$.

Morphological characters were extremely useful for identifying cultivars and, when used in combination with isozymes, have considerably increased cultivar discrimination. The mostimportant morphological characteristic of chestnuts for the market is the size of the nuts. It is also a useful character, as reported earlier, for the selection, management, and classification of chestnut cultivars (Oraguzie et al., 1988). Of all Spanish accessions, $58 \%$ produced more than 100 nuts $/ \mathrm{kg}$. Removing this low-value characteristic should be of high priority in breeding projects to produce new cultivars. Harvesting time is the second most important characteristic for marketing chestnuts. Yet only between $2 \%$ and $16 \%$ of the accessions from Andalucía, Asturias, and Castilla-León produced EAR nuts and in Galicia, LAT nuts are common (43\%). However, C. sativa tends to be harvested later than Asiatic species. Nut shape is important for distinguishing cultivars. Some cultivar names, such as Longal, are given based on shape (Pereira-Lorenzo, 1996a, and present data). Male catkin types and length of spines on burs are also useful characters to distinguish cultivars. We included in this study information on PDN, which is a disadvantage for nut peeling. In Spain up to 25\% of accessions, depending on region, had the detrimental character of producing more than $12 \%$ of PDN.

The Iberian Peninsula, in particular northern and northwestern Spain, is an important center of genetic diversity of chestnut, and a region where cultivars have been selected forcenturies. Material from this region is a valuable genetic resource for future chestnut breeding and selection.

\section{Literature Cited}

Aravanopoulos, F.A., A.D. Drouzas, and P.G. Alizoti. 2002. Electrophoretic and quantitative variation in chestnut (Castanea sativa Mill.) in Hellenic populations in old-growth natural and coppice stands. For. Snow Landscape Res. 76(3):429-434.

Bonnefoi, C. 1984. Etude du polymorphism enzymatique des populations forestières de chataignier, Castanea sativa Miller. PhD Diss., Université des Sciences et Techniques du Languedoc, Académie de Montpellier, Montpellier, France.
Breviglieri, N. 1955. Indagini ed osservazioni sulle migliori varietá italiane di Castagno. Centro di Studio Sul Castagno, Supplemento a la Ricerca Scientifica 2:27-164.

Dane, F. and L.K. Hawkins. 1999. Genetic variation and population structure of Castanea pumila var. ozarkensis. J. Amer. Soc. Hort. Sci. 124:666-670.

Fernández-Lamuño, J.A. 1984. Variedades del castaño como árbol frutal en el occidente de Asturias. Actas del Congreso Internacional del Castaño 133-150.

Goodman, M.M. 1972. Distance analysis in biology. Systematic Zoology 21:174-186.

Goodman, M.M. 1973. Genetic distances: Measuring dissimilarity among populations. Yrbk. Phys. Anthropology 17:1-38.

Goudet, J. 1995. Fstat version 1.2: A computer program to calculate Fstatistics. J. Hered. 86(6):485-486.

Goulao, L., T. Valdiviesso, C. Santana, and C.M. Oliveira. 2001. Comparison between phenetic characterisation using RAPD and ISSR markers and phenotypic data of cultivated chestnut (Castanea sativa Mill.). Genet. Resources Crop Evol. 48:329-338.

Huang, H., F. Dane, and T.L. Kubisiak. 1998. Allozyme and RAPD analysis of the genetic diversity and geographic variation in wild populations of the american chestnut (Fagaceae). Amer. J. Bot. 85(7):1013-1021.

International Union for the Protection of New Varieties of Plants (UPOV). 1988. Draft guidelines for the conduct of tests for distinctness, homogeneity and stability (CHESTNUT) TG/124/1 (proj.). UPOV, Geneva, Switzerland.

Kaufman, L. and P.J. Rousseeuw. 1990. Finding groups in data: An introduction to cluster analysis. Wiley, New York.

Oraguzie, N.C., D.L. McNeil, D.J. Klinac, R.D. Knowles, and J.R. Sedcole. 1998. Relationships of chestnut species and New Zealand chestnut selections using morpho-nut characters. Euphytica 99:27-33.

Pereira-Lorenzo, S., J. Fernandez-Lopez, and J. Moreno-Gonzalez. 1996a. Variability and grouping of northwestern Spanish chestnut cultivars (Castanea sativa). I. Morphological traits. J. Amer. Soc. Hort. Sci. 121(2):183-189.

Pereira-Lorenzo, S., J. Fernandez-Lopez, and J. Moreno-Gonzalez. 1996b. Variability and grouping of northwestern Spanish chestnut cultivars. II. Isoenzyme traits. J. Amer. Soc. Hort. Sci. 121(2):190-197.

Pereira-Lorenzo, S., B. Diaz-Hernandez, M. Ciordia-Ara, J. Ascasibar-Errasti, A.M. Ramos-Cabrer, and F. Sau. 2001a. Spanish chestnut cultivars. HortScience 36(2):344-347.

Pereira-Lorenzo, S., A.M. Ramos-Cabrer, D. Rios, A. Perdomo, and J. GonzálezPérez. 2001b. Update of the Spanish chestnut inventory of cultivars. Food and Agr. Org. of the United Nations, Centre International de Hautes Études Agronomiques Méditerranéennes-Nucis Nwsl. 10:34-37.

Pereira-Lorenzo, S., and A.M. Ramos-Cabrer. 2004. Chestnut, an ancient crop with future, p. 105-161. In: R. Dris and S.M. Jain (eds.). Production practices and quality assessment of food crops, Vol. 1. Preharvest practice. Kluwer Academic Publ., Dordrecht, The Netherlands.

Pigliucci, M., S. Benedettelli, and F. Villani. 1990a. Spatial patterns of genetic variability in Italian chestnut (Castanea sativa). Can J. Bot. 68:1962-1967.

Pigliucci, M., F. Villani, and S. Benedettelli. 1990b. Geographic and climatic factors associated with the spatial structure of gene frequencies in Castanea sativa Mill. from Turkey. J. Genet. 69(3):141-149.

Ramos-Cabrer, A.M. and S. Pereira-Lorenzo. 2005. Genetic relationship between Castanea sativa Mill. trees from northwestern to south Spain based on morphological traits and isoenzymes. Genet. Resources Crop Evol. 52(7):879-890.

Raymond, M. and F. Rousset. 1995. GENEPOP (version12). Population genetics software for exact tests and ecumenicism. J. Hered. 86:248-249.

Sawano, M., T. Ichii, T. Nakanishi, and Z. Kotera. 1984. Studies on identification of chestnut species and varieties by isozyme analysis. Sci. Rpt. Faculty Agr., Kobe University 16:67-71.

Sneath, P.H.A. and R.R. Sokal. 1973. Numerical taxonomy. Freeman, San Francisco.

Stilwell, K.L., H.M. Wilbur, C.R. Werth, and D.R. Taylor. 2003. Heterozygote advantage in the American chestnut, Castanea dentata (Fagaceae). Amer. J. Bot. 90(2):207-213.

Villani, F., S. Benedettelli, M. Paciucci, M. Cherubini, and M. Pigliucci. 1991a. Genetic variation and differentiation between natural populations of chestnut (Castanea sativa Mill.) from Italy, p. 91-103. In: Hattemer and Fineschi (eds.). Biochemical markers in the populations genetics of forest trees. SPB Academic Publ., The Hague, The Netherlands.

Villani, F., M. Pigliucci, S. Benedettelli, and M. Cherubini. 1991b. Genetic differentiation among Turkish chestnut (Castanea sativa Mill.) populations. Heredity 66:131-136.

Weir, B.S. and C.C. Cockerham. 1984. Estimating F-statistics for the analysis of population structure. Evolution 38:1358-1370.

Wright, S. 1951. The genetical structure of populations. Ann. Eugenics $15: 323-354$. 